\title{
LAS RECAÍDAS EN EL JUEGO PATOLÓGICO: UN ESTUDIO DE LAS SITUACIONES PRECIPITANTES
}

\author{
JAVIER FERNÁNDEZ-MONTALVO ${ }^{1}$, ENRIQUE ECHEBURÚA ${ }^{2}$ y CONCEPCIÓN BÁEZ ${ }^{3}$ \\ ${ }^{1}$ Universidad Pública de Navarra \\ ${ }^{2}$ Universidad del País Vasco \\ ${ }^{3}$ Centro de Salud Mental de Rentería
}

(Recibido el 18 de enero de 1999)

\begin{abstract}
En este artículo se presenta un estudio sobre las variables precipitantes de la recaída en una muestra de 21 jugadores patológicos de máquinas tragaperras, en el período de seguimiento de 1 año. Durante este tiempo los jugadores de la muestra sufrieron un total de 35 episodios de recaída. Los resultados obtenidos muestran que la mayor parte de las mismas tienen lugar en torno a los 3 primeros meses después de terminar el tratamiento. Las recaídas se producen, por orden de importancia, debido a un inadecuado manejo del dinero, a la presencia de estados emocionales negativos, al consumo de alcohol, al «craving» y a la presión social. Se comentan las implicaciones de este estudio para la investigación y la práctica clínica.
\end{abstract}

Palabras clave: Juego patológico. Recaída. Variables precipitantes.

Relapse in the pathological gambling: a Study of the associated situations

The aim of this paper was to determine the variables associated to relapse in a sample of 21 slot-machine pathological gamblers within an one year follow-up period. In this period there was a total of 35 relapse episodes. Results indicated that the most of relapses are observed in the first three months after treatment. The main reasons of relapse, in a hierarchic order, were the following ones: inadequate money management, negative emotional states, alcohol consumption, craving and social pressure. Implications of this study for clinical practice and future research in this field are commented upon.

Key words: Pathological gambling. Relapse. Variables associated to relapse.

\section{INTRODUCCIÓN}

Durante los últimos años ha cobrado especial importancia el estudio de las recaídas en las conductas adictivas. En este sentido su prevención constituye el principal reto en el tratamiento de las adicciones. De hecho, cada vez son más los estudios dirigidos a diseñar estrategias eficaces de intervención para conseguir el mantenimiento de la abstinencia (cfr. Casas y Gossop, 1993).

Agradecimientos: Este estudio se ha financiado con un proyecto de investigación de la Universidad del País Vasco (código n UPV 006.231-HA182/95).

Correspondencia: Javier Fenández Montalvo, Departamento de Psicología y Pedagogía, Universidad Pública de Navarra, Campus de Arrosadía, 31006 Pamplona. fernández.montalvo@si.unavarra.es.
En lo que se refiere al ámbito de la ludopatía, se ha avanzado considerablemente en esta dirección. Hoy en día se dispone de estrategias de eficacia contrastada para la intervención en prevención de recaídas (cfr. Echeburúa, Fernández-Montalvo y Báez, 1999a, en prensa; Fernández-Montalvo y Echeburúa, 1997).

Por otra parte, se ha producido un desarrollo importante en el conocimiento de los factores predictores. Así, por ejemplo, en el estudio de Báez, Echeburúa y Fernández-Montalvo (1995) las variables predictoras de recaída en una muestra de 48 jugadores patológicos de máquinas tragaperras fueron, por orden de importancia, la insatisfacción con el tratamiento, la menor antigüedad en la 
dependencia y, por último, el neuroticismo como dimensión de personalidad.

En otro estudio posterior de este mismo grupo ( $c f r$. Echeburúa, Fernández-Montalvo y Báez, 1999b, en prensa), con una muestra más amplia de 69 ludópatas adictos al mismo juego, las variables predictoras de fracaso terapéutico (recaída + abandono) fueron, en orden jerárquico, la insatisfacción con el tratamiento, el consumo abusivo de alcohol y el neuroticismo. Al figurar dos de estas variables (la insatisfacción con el tratamiento y el neuroticismo) en ambos estudios, se configuran como unas variables significativas $y$, por tanto, necesitadas de tener en cuenta en el tratamiento de este tipo de pacientes (cfr. Báez, Echeburúa y FernándezMontalvo, 1994; Fernández-Montalvo, Echeburúa y Báez, 1999, en prensa).

Otras variables predictoras de recaída encontradas en otros estudios son el bajo nivel de inteligencia (McCormick y Taber, 1991) y la mayor implicación en otras conductas adictivas en el pasado (no en el presente) (Zion, Tracy y Abell, 1991).

En definitiva, el avance experimentado en los últimos años en el diseño de estrategias eficaces de prevención de recaídas, así como en el conocimiento de las varia- bles predictoras de las mismas, ha sido, cuando menos, notable. No obstante, queda una parcela importante de este ámbito de las adicciones prácticamente sin estudiar: los factores precipitantes inmediatos de las recaídas en la ludopatía.

En este sentido, una revisión de los fracasos terapéuticos a largo plazo entre los alcohólicos, fumadores, heroinómanos, jugadores patológicos y obesos muestra unas tasas muy similares en estos diferentes cuadros clínicos: los dos tercios, aproximadamente, de todas las recaídas se producen en los tres primeros meses tras la terminación del tratamiento (Brown, 1987, 1989; Echeburúa y Corral, 1986; Marlatt y Gordon, 1985).

El fracaso en el mantenimiento de la abstinencia, independientemente del trastorno adictivo concreto, aparece frecuentemente asociado a las mismas tres situaciones de alto riesgo: estados emocionales negativos, conflictos interpersonales y presión social (Tabla 1). Estos datos apoyan la existencia de elementos comunes subyacentes en todas las conductas adictivas y, por ello, la justificación del estudio de la recaída como una variable relevante en sí misma (Echeburúa, 1993).

Tabla 1. Análisis de las situaciones que provocan recaídas en alcohólicos, fumadores, heroinómanos, jugadores y obesos (Marlatt y Gordon, 1985)

\begin{tabular}{|c|c|c|c|c|c|c|}
\hline Situación de la recaída & $\begin{array}{c}\text { Alcohólicos } \\
(N=70)\end{array}$ & $\begin{array}{c}\text { Fumadores } \\
(N=64)\end{array}$ & $\begin{array}{l}\text { Heroinomanos } \\
\quad(N=129)\end{array}$ & $\begin{array}{c}\text { Jugadores } \\
(N=29)\end{array}$ & $\begin{array}{l}\text { Obesos } \\
(N=29)\end{array}$ & $\begin{array}{c}\text { Total } \\
N=311\end{array}$ \\
\hline \multicolumn{7}{|l|}{ Determinantes intrapersonales } \\
\hline $\begin{array}{l}\text { Estados emocionales negativos } \\
\text { Estados físicos negativos } \\
\text { Estados emocionales positivos } \\
\text { Comprobar el control personal } \\
\text { Tentaciones y necesidades }\end{array}$ & $\begin{array}{r}38 \% \\
3 \% \\
\frac{9 \%}{11 \%}\end{array}$ & $\begin{array}{r}37 \% \\
2 \% \\
2 \% \\
\frac{5}{5} \%\end{array}$ & $\begin{array}{r}19 \% \\
9 \% \\
10 \% \\
2 \% \\
5 \%\end{array}$ & $\begin{array}{l}47 \% \\
\frac{-}{16 \%} \\
16 \%\end{array}$ & $\frac{33 \%}{\frac{3}{3} \%}$ & $\begin{array}{r}35 \% \\
3 \% \\
4 \% \\
5 \% \\
9 \%\end{array}$ \\
\hline TOTAL & $61 \%$ & $50 \%$ & $45 \%$ & $79 \%$ & $46 \%$ & $56 \%$ \\
\hline \multicolumn{7}{|l|}{ Determinantes interpersonales } \\
\hline $\begin{array}{l}\text { Conflictos interpersonales } \\
\text { Presion social } \\
\text { Estados emocionales positivos }\end{array}$ & $\begin{array}{r}18 \% \\
18 \% \\
3 \%\end{array}$ & $\begin{array}{r}15 \% \\
32 \% \\
3 \%\end{array}$ & $\begin{array}{r}14 \% \\
36 \% \\
5 \%\end{array}$ & $\begin{array}{c}16 \% \\
5 \% \\
-\end{array}$ & $\begin{array}{l}14 \% \\
10 \% \\
28 \%\end{array}$ & $\begin{array}{r}16 \% \\
20 \% \\
8 \%\end{array}$ \\
\hline TOTAL & $39 \%$ & $50 \%$ & $55 \%$ & $21 \%$ & $52 \%$ & $44 \%$ \\
\hline
\end{tabular}


Por lo que se refiere al juego patológico en concreto, los estados emocionales negativos constituyen el principal factor de riesgo. Si en las adicciones en general el $35 \%$ de las recaídas se asocian con un estado emocional negativo, en la ludopatía la tasa atribuible a este factor puede elevarse al $47 \%$ (Brown, 1989). Por otra parte, los conflictos interpersonales, especialmente los de tipo familiar (Lorenz, 1989), el reto del paciente consigo mismo sobre su capacidad de autocontrol, las tentaciones ambientales y las ganas experimentadas, así como, en menor medida, la presión social, constituyen otros factores importantes de peligro (cfr. Marlatt y Gordon, 1985).

El análisis de las recaídas en el juego patológico tiene un interés añadido en relación con el resto de los trastornos adictivos. Al no existir una sustancia tóxica que sea responsable de la dependencia física y explique, al menos hasta cierto punto, la probabilidad de recaída, en la ludopatía es posible estudiar los factores puramente psicológicos de tal fenómeno.

No obstante, excepto el estudio de Marlatt y Gordon (1985), que, por otra parte, no es específico del juego patológico, no existe ninguna otra investigación sobre los factores inmediatos que precipitan la vuelta al juego. Por ello, en este estudio se realiza un análisis detallado de las situaciones generadoras de recaída en los ludópatas.

\section{MÉTODO}

\section{Sujetos}

La muestra total de sujetos de este estudio contó con 21 jugadores patológicos que, después de haber recibido tratamiento con el objetivo de la abstinencia total en el Centro de Salud Mental de Rentería (Guipúzcoa) y en la Asociación de Ayuda a Ludópatas EKINTZA-DASA$L U D$ (Rentería, Guipúzcoa) entre los años 1990 y 1996, habían experimentado nuevos episodios de juego.

Los criterios para la selección de la muestra fueron los siguientes: a) haber recibido tratamiento por un problema de ludopatía en uno de los centros anteriormente mencionados; b) haber permanecido abstinente del juego durante un tiempo mínimo de un mes (requisito necesario para hablar propiamente de recaída); c) haber experimentado uno o más episodios de juego una vez transcurrido este tiempo.

En concreto, los 21 pacientes que forman parte de este estudio habian sufrido 35 episodios de recaída. Estos sucesos incluían tanto una caída aislada como una recaída total en el juego.

\section{Procedimiento}

Los pacientes de este estudio forman parte de dos investigaciones más amplias sobre el tratamiento de la ludopatía (cfr. Echeburúa, Báez y Fernández-Montalvo, 1996; Echeburúa et al., 1999a). En ellas se llevaron a cabo evaluaciones repetidas en los seguimientos de 1, 3, 6 y 12 meses. En cada episodio de recaída los terapeutas evaluaban las situaciones concretas que habían provocado la vuelta al juego, así como el tiempo transcurrido desde la finalización del tratamiento.

En una fase posterior se procedió a la agrupación de los motivos de recaída en categorías más amplias.

\section{RESULTADOS}

Tiempo transcurrido desde la finalización del tratamiento hasta la primera recaída

En la Tabla 2 se presentan los datos relacionados con la primera recaída de 
Tabla 2. Momento de la primera recaída

\begin{tabular}{lcccc}
\hline $\begin{array}{l}\text { Momento de la } \\
\text { Primera recaída }\end{array}$ & $N$ & $(\%)$ & \multicolumn{2}{c}{ Frecuencia acumulada } \\
\hline 1 mes & 8 & $(38,1 \%)$ & 8 & $(\%)$ \\
3 meses & 6 & $(28,6 \%)$ & 14 & $(38,1 \%)$ \\
6 meses & 5 & $(23,8 \%)$ & 19 & $(66,7 \%)$ \\
12 meses & 2 & $(9,5 \%)$ & 21 & $(100 \%)$ \\
\hline
\end{tabular}

cada paciente, así como el momento en que ésta se detectaba.

Como se aprecia en la tabla, las dos terceras partes de todas las recidivas -en concreto, el 66,7\%- se producen en los tres primeros meses después de terminar el tratamiento. En el seguimiento de los 6 meses ya se han producido el $90,5 \%$ de las mismas. Las recaídas a partir de este momento son ya aisladas en los casos tratados con éxito.

Análisis de las situaciones que provocan recaídas en los jugadores patológicos

Para realizar estos análisis se cuenta con un número total de 35 episodios de recaída. Conviene señalar que algunos pacientes habían sufrido más de un episodio de juego.

En este sentido, los principales factores precipitantes de la recaída fueron, por orden de importancia, los siguientes: el manejo inadecuado del dinero, los estados emocionales negativos, el consumo abusivo de alcohol, la avidez por el juego (el «craving») y, por último, la presión social (Tabla 3 ).

Tabla 3. Factores precipitantes de la recaída en los jugadores patológicos $(\mathrm{N}=35)$

\begin{tabular}{lrc}
\hline Factores precipitantes & $N$ & $(\%)$ \\
\hline Manejo inadecuado del dinero & 17 & $(48,6 \%)$ \\
Estados emocionales negativos & 9 & $(25,7 \%)$ \\
Consumo abusivo de alcohol & 5 & $(14,3 \%)$ \\
Avidez por el juego ( (craving») & 2 & $(5,7 \%)$ \\
Presión social & 2 & $(5,7 \%)$ \\
\hline
\end{tabular}

En concreto, casi la mitad de todas las recaídas se producen por un control inapropiado del dinero (disponibilidad de unos ingresos económicos inesperados, vigilancia excesiva del dinero por parte de la familia, terminación del pago de las deudas, etc.). El segundo factor en importancia es la presencia de estados emocionales negativos (aburrimiento/tristeza, ansiedad/estrés, ira/discusiones, etc.), que explica el $25 \%$ de las recaídas. El resto de categorías (consumo abusivo de alcohol, "craving» y presión social), si bien son también importantes, explican un porcentaje mucho menor de los fracasos terapéuticos.

\section{CONCLUSIONES}

En esta investigación se han analizado las situaciones precipitantes de la recaída en los jugadores patológicos de máquinas tragaperras que acuden a tratamiento. Como se ha señalado anteriormente, en el ámbito de la ludopatía estos factores son realmente desconocidos, puesto que solamente existe un estudio -y éste es inespecífico- dirigido a tal fin (cfr. Marlatt y Gordon, 1985).

Según los resultados obtenidos, la mayor parte de las recaídas se producen en torno a los 3 primeros meses después de terminar el tratamiento. Además, en el seguimiento de los 6 meses se han producido ya la práctica totalidad de las recaídas estudiadas. Estos datos coinci- 
den con los obtenidos por Marlatt y Gordon (1985), tanto en lo que se refiere al juego patológico como al resto de las conductas adictivas. Por lo tanto, los primeros meses de seguimiento, una vez terminada la intervención terapéutica propiamente dicha, constituyen un momento crítico para el mantenimiento del cambio conductual conseguido con la terapia.

De este modo, los programas de prevención de recaídas, aplicados una vez conseguida la abstinencia, pueden ser de gran importancia para el mantenimiento de la misma. Este tipo de intervenciones, en las que se prepara al paciente para afrontar las situaciones críticas de la vida cotidiana cuando ya se ha terminado el tratamiento, pueden reducir significativamente el número de recaídas en los primeros meses de seguimiento. No se debe olvidar que los jugadores patológicos presentan numerosas distorsiones cognitivas (cfr. Fernández-Montalvo, Báez y Echeburúa, 1996), que perduran habitualmente más allá de la intervención terapéutica y que pueden facilitar este tipo de fracasos iniciales. Por ello, en el reciente estudio de Echeburúa et al. (1999a) se puso precisamente a prueba la eficacia de dos modalidades de prevención de recaídas -individual y grupal-, en relación con un grupo de control. Los resultados obtenidos en el seguimiento de los 12 meses mostraron un número significativamente menor de fracasos en los jugadores que habían recibido la intervención para la prevención de recaídas.

Desde otra perspectiva, en este estudio la mayor parte de las recaídas se producen, por orden de importancia, debido a un inadecuado manejo del dinero, a la presencia de estados emocionales negativos, al consumo abusivo de alcohol, al "craving" y a la presión social. Tres de estas variables - los estados emocionales negativos, el «craving» o necesidad subjetiva de jugar y la presión social - coin- ciden con las obtenidas por Marlatt y Gordon (1985). No obstante, resulta sorprendente que en esta investigación sea el control inapropiado del dinero el principal factor asociado a la recaída y que, sin embargo, esta variable, tan característica del juego patológico, no aparezca en la investigación clásica de Marlatt y Gordon (1985).

La experiencia clínica de los autores de este texto en el ámbito de la ludopatía constata las enormes dificultades de este tipo de pacientes para manejar adecuadamente el dinero, así como el riesgo que ello supone para la posterior recaída. De hecho, esta variable explica casi la mitad de los episodios estudiados (el $48,6 \%$ ). En este sentido, las principales situaciones de riesgo observadas son la disposición de un dinero extra - por ejemplo, cobrar en mano unos atrasos de la empresa-, el control excesivo del dinero por parte de la familia, que lleva al jugador a un hastío prematuro del control de estímulos, y la terminación del pago de las deudas, con el sentimiento de liberación y de desahogo que ello supone. Todas estas situaciones relacionadas con el manejo del dinero facilitan los episodios de descontrol. Por ello, es fundamental que los programas de prevención de recaídas en el ámbito del juego patológico incluyan el aprendizaje de habilidades de administración del dinero.

Por otra parte, otro aspecto que tampoco aparece reflejado en la investigación de Marlatt y Gordon (1985) es el consumo abusivo de alcohol. En concreto, el $14,3 \%$ de las recaídas de este estudio se deben a este factor. Una posible explicación de la importancia de esta variable deriva de que el consumo de alcohol dificulta los dos elementos fundamentales para prevenir las recaídas: la identificación de las situaciones de alto riesgo y la puesta en práctica de las estrategias de afrontamiento necesarias para superar con éxito esa situación. Los niveles ele- 
vados de ingesta alcohólica, al disminuir la capacidad atencional del sujeto, dificultan el reconocimiento e identificación de una situación concreta como peligrosa. Un ex jugador que ha bebido en exceso puede ser incapaz de discriminar las verdaderas circunstancias amenazantes de las que no lo son y verse así mermado en las posibilidades de afrontar con éxito una situación de este tipo.

Además, el consumo de alcohol puede inhibir la puesta en práctica de las habilidades de afrontamiento necesarias. Así, un sujeto que ha bebido en exceso puede saber reconocer las situaciones de alto riesgo e identificarlas como peligrosas, pero sentirse bloqueado para la ejecución de las respuestas apropiadas para afrontar esa situación.

En cuanto a los estados emocionales negativos - segunda variable en importancia en este estudio y primera en el de Marlatt y Gordon (1985)-, las emociones pueden ser muy variadas (aburrimiento, estrés, frustración, discusiones, ansiedad, tristeza, etc.) y explican el $25 \%$ de las recaídas. No se debe olvidar que el neuroticismo es un predictor de recaídas en el juego patológico (Báez et al., 1995; Echeburúa et al., 1999b) y que, además, aparece sobrerrepresentado entre los jugadores (Báez et al., 1994; FernándezMontalvo et al., 1999). En este sentido, una alteración emocional dificulta, al igual que ocurre con el consumo de alcohol, el comportamiento racional del paciente, por lo que se merma la capacidad de afrontar adecuadamente las situaciones de riesgo. El jugador, en este tipo de situaciones, recuerda sesgadamente las consecuencias positivas a corto plazo de la conducta adictiva y se implica, como medio de liberar la tensión - una forma inadecuada de autoterapia-, en la conducta de juego. Por ello, cobra una especial importancia el aprendizaje de estrategias de afrontamiento de los estados emocionales adversos - habilidades de comunicación, técnicas de solución de problemas, relajación, actividades de tiempo libre, etc.- que impidan el recurso «fácil» a la conducta de juego.

Por último, la presión social (propuestas de jugar a medias, incitación al juego, etc.) y el "craving» (avidez por el juego, ganas de jugar, ver a alguien jugar y no poder resistirse, etc.), que explican casi el $12 \%$ de las recaídas entre uno y otro, son dos aspectos importantes a tener en cuenta. En este sentido, el aprendizaje de estrategias de autocontrol, así como de las habilidades de comunicación adecuadas para afrontar las incitaciones al juego, puede aumentar las expectativas de autoeficacia y reducir, por lo tanto, los fracasos debidos a este tipo de factores.

En cualquier caso, el proceso de recaída está vinculado con determinantes situacionales que varían de unas adicciones a otras. Más allá de los factores comunes presentes en todas las conductas adictivas -estados emocionales negativos, presión social y conflictos interpersonales-, existen variables específicas a cada adicción. Por ello, una línea de investigación interesante para los próximos años es la adaptación de los programas generales de prevención de recaídas a las situaciones de riesgo características de cada adicción, así como a las diferencias individuales de los pacientes. Por otra parte, otro ámbito de estudio futuro debe encaminarse a intentar determinar qué relación psicológica se establece entre el primer episodio y el descontrol posterior en la recaída.

\section{REFERENCIAS BIBLIOGRÁFICAS}

Báez, C., Echeburúa, E. y Fernández-Montalvo, J. (1994). Características demográficas, de personalidad y psicopatológicas de los jugadores patológicos de máquinas tragaperras en tratamiento: un estudio descriptivo. Clínica y Salud, 5, 289-305. 
Báez, C., Echeburúa, E. y Fernández-Montalvo, J. (1995). Variables predictoras de abandonos y recaídas en el tratamiento del juego patológico. Análisis y Modificación de Conducta, 21, 5-22.

Brown, R.I.F. (1987). Dropouts and continuers in Gamblers Anonymous: Part 2. Analysis of free-style accounts of experiences with G.A. Journal of Gambling Behavior, 3, 68-79.

Brown, R.I.F. (1989). Relapses from a gambling perspective. En M. Gossop (Ed.), Relapses and addictive behaviour. London: Croom Helm.

Casas, M. y Gossop, M. (Eds.) (1993). Recaída y prevención de recaídas. Barcelona: Citrán.

Echeburúa, E. (1993). Las conductas adictivas: ¿una ruta común desde el "crack» al juego patológico?. Psicología Conductual, 1, 321-337.

Echeburúa, E., Báez, C. y Fernández-Montalvo, J. (1996). Comparative effectiveness of three therapeutic modalities in the psychological treatment of pathological gambling: long-term outcomes. Behavioural and Cognitive Psychotherapy, 24, 51-72.

Echeburúa, E. y Corral, P. (1986). Predicción de la recaída en las conductas adictivas: estrategias de intervención. Drogalcohol, 11, 16-24.

Echeburúa, E., Fernández-Montalvo, J. y Báez, C. (1999a, en prensa). Relapse prevention in the treatment of pathological gambling: long-term outcome. Behavior Therapy.

Echeburúa, E., Fernández-Montalvo, J. y Báez, C. (1999b, en prensa). Predictors of therapeutic failure in pathological gamblers following behavioral treatment. Behavior Modification.

Fernández-Montalvo, J., Báez, C. y Echeburúa, E. (1996). Distorsiones cognitivas de los jugadores patológicos de máquinas tragaperras en tratamiento: un estudio descriptivo. Cuadernos de Medicina Psicosomática y Psiquiatría de Enlace, 37, 13-23. Fernández-Montalvo, J. y Echeburúa, E. (1997). Manual práctico del juego patologico. Ayuda para el paciente y guia para el terapeuta. Madrid. Pirámide.

Fernández-Montalvo, J., Echeburúa, E. y Báez, C. (1999, en prensa). Variables de inteligencia y de personalidad de los jugadores patológicos de máquinas tragaperras: un estudio descriptivo. Psicología Conductual.

Lorenz, V.C. (1989). Some treatment approaches for family members who jeopardize the compulsive gambler's recovery. Journal of Gambling Behavior, 5, 303-312.

Marlatt, G.A. y Gordon, J.R. (1985). Relapse prevention: maintenance strategies in addictive behavior change. New York: Guilford Press.

McCormick, R.A. y Taber, J.I. (1991). Followup of male pathological gamblers after treatment: the relationship of intellectual variables to relapse. Journal of Gambling Studies, 7, 99-108.

Zion, M.M., Tracy, E. y Abell, N. (1991). Examining the relationship between spousal involvement in Gam-Anom and relapse behaviors in pathological gamblers. Journal of Gambling Studies, 7, 117-131. 